\title{
Fatores relacionados ao risco de Doença Arterial Coronariana entre estudantes de enfermagem
}

\author{
Associated risk factors for Coronary Heart Disease among nursing students \\ Factores relacionados al riesgo de Enfermedad Coronaria entre estudiantes de enfermería
}

\begin{tabular}{c} 
Alessa Alves', Isaac Rosa Maroues' \\
'Universidade de Santo Amaro. Faculdade de Enfermagem. São Paulo, SP \\
\hline
\end{tabular}

Submissão: 20/08/2009

Aprovação: 01/12/2009

\section{RESUMO}

Um estudo correlacional foi realizado com 303 alunos de curso de graduação em enfermagem de uma universidade privada da cidade de São Paulo. O estudo objetivou estabelecer associações/correlações entre fatores de risco para Doença Arterial Coronariana. O fator de risco isolado de maior relevância foi o sedentarismo $(80,2 \%)$. Os fatores de risco associados de maior significância foram IMC $\geq 25$, correlacionado com a faixa etária dos 21 a 25 e o gênero masculino $(p=0,043)$. Concluiu-se que entre os estudantes de enfermagem os hábitos alimentares e a falta de atividade física são determinantes para o sobrepeso e Que estes fatores podem estar associados à ocorrência futura de Doença Arterial Coronariana.

Descritores: Doença das coronárias; Risco atribuível; Estudantes de enfermagem.

\section{ABSTRACT}

A correlational study was carried out with 303 nursing students from a private nursing school in the city of São Paulo, SP, Brazil. The study aimed at establishing correlation among risk factors for Coronary Heart Disease. Isolated risk factor of major relevance was sedentary lifestyle $(80,2 \%)$. Associated risk factors of major significance was BSA $\geq 25$ correlated with age range from 21 to 25 and gender male $(p=0,043)$. It was concluded that among nursing students the feeding habits and sedentary lifestyle are determinant for weight overload and this factors may be associated to a future occurrence of Coronary heart Disease.

Descriptors: Coronary disease; Attributable risk; Nursing students.

\section{RESUMEN}

Un estudio correlacional fue realizado con 303 estudiantes de grado en enfermería en una universidad privada en São Paulo, SP, Brasil. El studio objetivó establecer asociaciones/correlaciones entre factores de riesgo para la Enfermedad Coronaria. El factor de riesgo aislado de mayor relevancia fue el sedentarismo $(80,2 \%)$. Los factores de riesgo asociados de mayor significancia fueran el IMC $\geq 25$ correlacionado con la edad de los $2 \mathrm{I}$ a los 25 años y el género masculino $(p=0,043)$. Se hay concluido Que entre los estudiantes de grado en enfermería los hábitos de alimentación y la inactividad física son determinantes para el sobrepeso y que estos factores pueden estar asociados a futura ocurrencia de la Enfermedad Coronaria.

Descriptores: Enfermedad coronaria; Riesgo atribuible; Estudiantes de enfermería. 


\section{INTRODUÇÃO}

A Doença Arterial Coronariana (DAC) ou aterosclerose coronariana é caracterizada pelo estreitamento das artérias coronárias em decorrência do espessamento endotelial causado pelo acúmulo de placas ateromatosas. Os efeitos da DAC traduzem-se em episódios de dor anginosa Quando o portador da doença expõe-se a fatores precipitantes $^{(1)}$.

A expressão "cardiopatia is@uêmica" designa uma afecção cardíaca Que tem como característica principal a isquemia miocárdica. Cerca de $90 \%$ dos casos são decorrentes de aterosclerose coronária, justificando nessas condições a denominação de cardiopatia aterosclerótica ou DAC. Vale ressaltar, contudo, Que as expressões cardiopatia isQuêmica, doença arterial coronariana e cardiopatia aterosclerótica não são exatamente sinônimas ${ }^{(1)}$.

A DAC é a causa mais comum de morte nos países desenvolvidos e em desenvolvimento em todo o mundo. No Brasil, a mortalidade relacionada a DAC oscila entre 11,3 a 2,5 óbitos por 100.000 habitantes, dependendo de fatores locais. O Rio de Janeiro é o estado Que apresenta o maior índice ${ }^{(2)}$. Entretanto, existe declínio nestes índices nos últimos anos.

Em geral, as manifestações clínicas da DAC, como Infarto do Miocárdio, Acidente Vascular Encefálico e Doença Vascular Periférica, têm início a partir da meia idade. No entanto, estudos indicam que o processo aterosclerótico começa a se desenvolver na infância. Estrias gordurosas, precursoras das placas ateroscleróticas, começam a aparecer na camada íntima da aorta aos três anos de idade e nas coronárias durante a adolescência, podendo progredir significativamente na terceira e Quarta décadas de vida ${ }^{(3-5)}$.

Com a evolução das tecnologias e conhecimentos nas áreas de humanas e saúde, a perspectiva de vida dos seres humanos tem sido cada vez mais alta, com essa elevação a propensão para problemas de saúde se acentua em nossa sociedade.

Em 1948, após a II Guerra mundial, Quando se verificou o aumento da mortalidade por DAC, iniciou-se o maior estudo epidemiológico sobre as doenças cardíacas, o "Framingham Heart Study". Inicialmente, foram identificados como os principais fatores de risco cardiovasculares: hipertensão arterial sistêmica, hipercolesterolemia, tabagismo, obesidade, diabetes mellitus e sedentarismo. Posteriormente, foram descobertos outros fatores como hipertrigliceridemia, redução dos níveis de HDL-colesterol (HDL-c), idade, sexo e fatores psicossociais. Esse estudo observou maior prevalência de DAC no sexo masculino, na raça branca e, nas Mulheres, um aumento de incidência após a menopausa ${ }^{(6,7)}$.

Segundo a Organização Mundial da Saúde (OMS) as doenças cardiovasculares são responsáveis por $30 \%$ do total de mortes no mundo. A projeção da OMS é Que esse grupo de doenças seja a primeira causa de morte em todos os países em desenvolvimento até $2010^{(8)}$.

Hoje, não seria surpreendente encontrar uma população de pacientes com características diferentes das décadas anteriores e com perfil diferente da doença com relação a tratamento e evolução da doença.

\section{REVISÃO DA LITERATURA}

Devido à impossibilidade de identificar com certeza Quem desenvolverá uma síndrome isQuêmica consequente de uma lesão aterosclerótica, existem alguns fatores de risco diretamente relacionados com a progressão e as complicações da lesão aterosclerótica. Estes fatores de risco são igualmente importantes em ambos os sexos, apesar da importância de ser relativamente maior para um determinado grupo. A associação entre eles tem efeito cumulativo; isto é, Quanto maior o número e intensidade dos fatores de risco, maior a incidência das doenças cardiovasculares. Atualmente, sugere-se que mais de $80 \%$ dos casos de morte por doenças cardiovasculares estejam associadas a fatores de risco já $\operatorname{conhecidos}^{(9)}$.

A valorização dos chamados fatores de risco, foi uma das grandes mudanças na maneira de encarar a DAC. Isto nada mais é do Que a transposição para a cardiologia do conceito de multicausalidade das doenças. lá foram reconhecidos mais de 21 fatores de risco. Porém, muita distância existe entre o conhecimento destes fatores de risco e a influência Que os mesmos têm entre si no sentido de se atribuir como causa para a DAC(1).

Os fatores de risco pedem ser divididos em modificáveis e não modificáveis. Quanto maior o número de fatores de risco presentes, maior a chance de apresentar um evento cardiovascular. Os fatores de risco não-modificáveis estão relacionados à herança genética de um indivíduo e deixam Questões em aberto com relação à DAC. Mesmo sabendo que não se pode alterar a herança genética de um indivíduo, existe a possibilidade de alterar seu estilo de vida, reduzindo a possibilidade de ter um evento cardíaco precocemente ${ }^{(1)}$.

Os fatores de risco não-modificáveis também incluem o sexo ou gênero e sua incidência de morbi-mortalidade é maior e mais precoce no sexo masculino. Havendo, porém, uma diminuição desta discrepância Quando relacionados a mulheres pós menopausa ${ }^{(7)}$. Em mulheres, as manifestações clínicas aparecem em média cerca de 10 a 15 anos mais tardiamente Que os homens, fato este Que possivelmente é explicado pela proteção estrogênica. De fato, a DAC ocorre de duas a três vezes mais em mulheres após a menopausa do que aquelas na pré-menopausa. Estudos das décadas de 50 e 60 descreveram Que a menopausa precoce estava associada a aumento de DAC ${ }^{(10)}$.

Outro fator é a idade, a Qual devido a mudanças hemodinâmicas e anatômicas do sistema cardiovascular levam a uma disfunção arterial progressiva mesmo em pessoas normais ${ }^{(7)}$.

Outro fator não-modificável é o antecedente familiar. Considerase este fator Quando o indivíduo tem familiares diretos como pai, mãe e irmãos Que apresentaram a doença antes dos 55 anos de idade. Relacionado a este fator é necessário ressaltar o papel dos fatores genéticos, os Quais por não serem modificáveis, não recebem a mesma atenção nas pesquisar epidemiológicas por um nítido interesse comercial por parte das indústrias farmacêuticas ${ }^{(1)}$. Presença de histórico familiar evidencia maior probabilidade de desenvolver $\mathrm{DAC}^{(1,7)}$

Por outro lado, os fatores de risco modificáveis, são os Que mais podem receber a atenção no sentido das intervenções preventivas. O tabagismo é o principal fator modificável elevador da probabilidade de morbidade e mortalidade na DAC e em doenças neurocardiovasculares, é também a principal causa de morte relativa a outras patologias que se pode prevenir. A magnitude do problema é identificada ao se considerar a estimativa da OMS de Que cerca de $1 / 3$ da população mundial adulta seja fumante ${ }^{(1 I)}$. Os malefícios 
do cigarro estão diretamente ligados à Quantidade e tempo de consumo. É notável a diminuição da prevalência de DAC em homens Que reduzem o consumo de cigarros. E o consumo de cigarros reduz a relativa proteção a DAC no sexo feminino ${ }^{(7)}$.

A Hipertensão Arterial Sistêmica (HAS) é considerada como o mais importante fator de risco para o desenvolvimento não somente da DAC como para todas as doenças isquêmicas incluindo o Acidente Vascular Encefálico ${ }^{(9)}$. Em indivíduos hipertensos o risco para doenças cardíacas é Quatro vezes maior entre as mulheres e três vezes entre os homens, Quando comparadas com indivíduos normotensos. Nas mulheres o risco para hipertensão é consideravelmente maior principalmente nos últimos três meses de gravidez, e no pós menopausa ${ }^{(7)}$.

Outro fator de risco modificável é a Diabetes Mellitus (DM). Em portadores de DM as doenças cardiovasculares são responsáveis por aproximadamente $80 \%$ dos óbitos ${ }^{(7)}$.

A dislipidemia é a condição na Qual há concentrações anormais de lipídios ou lipoproteínas no sangue. Trata-se de um fator de risco importante para o desenvolvimento de complicações da aterosclerose. Em diferentes populações, estão bem estabelecidas as correlações entre o risco para DAC e concentrações séricas elevadas de colesterol total (CT), particularmente de lipoproteínas de baixa densidade (LDL-C), assim como concentrações reduzidas de lipoproteínas de alta densidade (HDL-C) ${ }^{(12,13)}$.

O sedentarismo, Que é a falta de atividade física regular, assume um papel extremamente importante como fator de risco para DAC. Estudos comprovam que a atividade física aeróbica de moderada intensidade Quando realizada de forma regular, no mínimo 30 minutos ao dia pelo menos 3 vezes na semana pode atingir impacto na redução do risco de evento cardiovascular na ordem de $30 \%$ a $40 \%{ }^{(10)}$.

O sobrepeso é outro fator de risco importante para o desenvolvimento de DAC. Aproximadamente $32 \%$ da população brasileira apresenta sobrepeso, ou seja: Índice de Massa Corporal (IMC) $\geq 25$, sendo esta taxa de $38 \%$ para o sexo feminino e de $27 \%$ para o sexo masculino. A obesidade (IMC > 30) foi encontrada em $8 \%$ da população brasileira ${ }^{(14)}$.

A palavra estresse tornou-se de uso corriqueiro. Usa-se como sendo a causa ou a explicação para inúmeros acontecimentos Que afligem a vida humana moderna ${ }^{(15)}$. O estresse está diretamente ligado ao estilo de vida adotado pelo indivíduo. Na verdade, não é fácil definir o Que seja estilo de vida, pois depende de fatores intrínsecos e extrínsecos como os socioeconômicos e culturais ${ }^{(1,15)}$.

A incidência destes riscos em diversas populações específicas tem sido estudada. Coelho et al ${ }^{(16)}$, estudaram o perfil lipídico entre estudantes de medicina e verificaram Que há significância entre o IMC elevado associado ao perfil lipídico como fator de risco para DCV. Outro estudo ${ }^{(17)}$, investigou relações dos fatores entre estudantes de nutrição com resultados semelhantes. Nenhum estudo nacional com estudantes de enfermagem foi localizado na revisão da literatura.

Os enfermeiros, pelo conhecimento e acesso privilegiado a informações sobre saúde, são vistos em nossa sociedade como exemplo de comportamento a ser seguido pelos leigos e seu papel de educador e exemplo é de grande impacto para sociedade Que os cerca.

Por esta razão, este estudo teve como objetivo identificar o perfil de risco para Doença Arterial Coronariana entre estudantes de um curso de graduação em enfermagem de uma universidade privada da zona sul da cidade de São Paulo.

\section{METODOLOGIA}

Tratou-se de uma pesquisa com desenho não-experimental do tipo correlacional ${ }^{(18)}$. A projeto de peseuisa foi aprovado pelo Comitê de Ética em Pesquisa da Universidade de Santo Amaro, Protocolo ${ }^{\circ}$ 092/2009.

A peseuisa foi realizada com alunos matriculados do curso de graduação em enfermagem da referida universidade. A população compreendeu os alunos dos turnos matutino e vespertino regularmente matriculado no segundo semestre letivo de 2009.

A amostra foi composta pela técnica não-probabilística de conveniência; ou seja, participaram da pesquisa os alunos Que, após a leitura da Carta de Informação, consentiram pela participação mediante a assinatura do Termo de Consentimento Livre e Esclarecido.

A coleta de dados foi realizada durante o mês de agosto de 2009. Foi utilizado um Questionário com perguntas semiestruturadas, contendo duas partes: dados de identificação e os dados sobre os fatores de risco. A aplicação deste Questionário ocorreu em sala de aula com 303 alunos. Foram excluídos do estudo os Questionários com preenchimento incompleto.

As variáveis de identificação da amostra incluíram: sexo, idade, semestre Que cursa atualmente, período. As variáveis relativas aos fatores de risco para DAC incluíram: IMC, raça, histórico pessoal de Hipertensão Arterial Sistêmica (HAS) e Diabetes Mellitus (DM), prática de atividade física e padrão de sono, prevalência de estresse e tabagismo. Ainda foram avaliadas as variáveis: Realização de dosagem de colesterol e triglicérides e a presença de história familiar para DAC.

Para a análise dos resultados foram aplicados os seguintes testes ${ }^{(19)}$ : teste de $\mathrm{G}$ de Cochran, o Qual teve como objetivo estudar as ocorrências simultâneas dos fatores de risco para DAC. Este teste foi aplicado em separado para gêneros femininos ou masculinos, nos grupos de IMC $\leq 25$ e IMC $\geq 25$. Em seguida foi realizado o teste de Qui-Quadrado para comparar as distribuições etárias de mulheres e de homens nos grupos de IMC $\leq 25$ ou IMC $\geq 25$. Posteriormente, foi aplicado teste exato de Fisher, com a finalidade de comparar os gêneros feminino e masculino em relação a presença de HAS, separando-se por grupos em IMC $\leq 25$ e IMC $\geq 25$. O nível de seeüência foi fixado em 0,05 ou $5 \%$.

\section{RESULTADOS}

A partir da coleta de dados realizada em Setembro de 2009 , participaram do estudo $303(46,2 \%)$ alunos de um total de 655 alunos matriculados, sendo Que 16 Questionários foram excluídos devido estarem incompletos ou preenchidos erradamente.

As amostras foram de estudantes desde o $1^{\circ}$ até o $8^{\circ}$ semestre dos horários matutino $(n=105 / 34,7 \%)$ e noturno $(n=198 /$ $65,3 \%)$. No $1^{\circ}$ semestre $(n=20 / 6,6 \%), 2^{\circ}$ semestre $(n=45 /$ $14,9 \%), 3^{\circ}$ semestre $(n=20 / 6,6 \%), 4^{\circ}$ semestre $(n=110 / 36,3 \%)$, $5^{\circ}$ semestre $(n=36 / 11,9 \%), 6^{\circ}$ semestre $(n=34 / 11,2 \%), 8^{\circ}$ semestre $(n=38 / 12,5 \%)$. Não havendo coleta de dados entre os graduandos $7^{\circ}$ semestre por inacessibilidade destes, devido atividades externas à instituição no período da coleta de dados. Quanto 
ao gênero, houve predominância do sexo feminino em todas as faixas etárias (feminino $n=255 / 84,2 \%$ e masculino $n=48 / 15,8 \%$ ).

De acordo com dados antropométricos, foram obtidos os valores de IMC, Que foi em média $\geq 25$ em 26,4\% da amostra estudada. Conforme os dados da Figura I, verifica-se Que houve uma distribuição uniforme de IMC $\geq 25$ em todas as faixas etárias, considerando Que nas faixas etárias mais altas (36 a 40 e $\geq 4$ l anos) a representatividade de estudantes é menor. $E$ nestas faixas houve equilíbrio entre IMC $\leq 25$ e $\geq 25$.

Quanto à raça/etnia, na soma entre homens e mulheres, houve predomínio da raça branca $(n=159 / 52,5 \%)$, seguido dos pardos $(n=103 / 34,0 \%)$, posteriormente os negros $(n=35 / 11,6 \%)$, e em menor Quantidade os amarelos $(n=06 / 2,0 \%)$.

Quanto à HAS, houve presença na minoria dos participantes da pesquisa $(n=11 / 3,6 \%)$ entre homens e mulheres (Tabela 1). A correlação entre os sexos não foi significativa $(p=0,214$ e 0,3083 respectivamente).

A incidência de DM foi considerada baixa Quando associado ao risco de DAC, com apenas um $(n=1)$ estudante do sexo feminino $(0,03 \%)$; o que se pode considerar um fator de pouca relevância para esta amostra.

O sedentarismo foi observado na maioria dos graduandos. Foram identificados 243 (equivalente a 80,2\%) de sedentários, contra 60 não sedentários (Que ptraticam alguma ativiade física), o Que equivale a $19,8 \%$.

O tabagismo não obteve relevância conforme sexo e idade, com o total de 20 fumantes, o Que equivale a $6,6 \%$ da amostra estudada, com média de consumo de 7,4 cigarros por dia (valor obtido através da divisão do total de cigarros/dia citados por todos os fumantes, divididos pelos mesmos).

Sobre estes resultados, há Que se destacar que a atividade física é o fator de risco de maior prevalência para ambos os gêneros, independentemente do valor do IMC $(<25$ ou $\geq 25)$. Entre os participantes da pesquisa, 89,4\% (271) têm mais de um fator de risco. O número maior de fatores de risco encontrado individualmente foi de oito fatores, Dados isolados sobre os fatores de risco e a correlação com o IMC estão apresentados na Tabela 2. De acordo com o Teste G de Cochran, não houve significância para nenhum dos fatores de risco ao serem consideradas as associações entre todos os fatores de risco entre si.

Ao realizar a associação entre a idade e o IMC $\geq 25$, foi verificado Que esta associação representou um valor significante como probabilidade para o desenvolvimento de DAC. Entre os estudantes do gênero masculino da faixa etária de 21 a 25 anos houve uma associação com o IMC $\geq 25$ significante $(p=0,043)$. Em seguida, os estudantes do gênero masculino da faixa etária de 26 a 30 anos uma associação significante de 40,0\% $(p=0,034)$. Entre os estudantes do gênero feminino com IMC $\geq 25$ na faixa etária 3 I a 40 anos, houve uma associação significante de $26,3 \%(p=0,01$ l $)$. Em termos gerais, entre os estudantes com IMC $\geq 25$, uma significância de 25,0\% $(p=0,025)$. Maiores detalhes estão apresentados na Tabela 3 .

Quanto à dosagem de colesterol, 64,0\% dos estudantes já realizaram ao menos uma avaliação, eneuanto $36 \%$ não o realizaram.

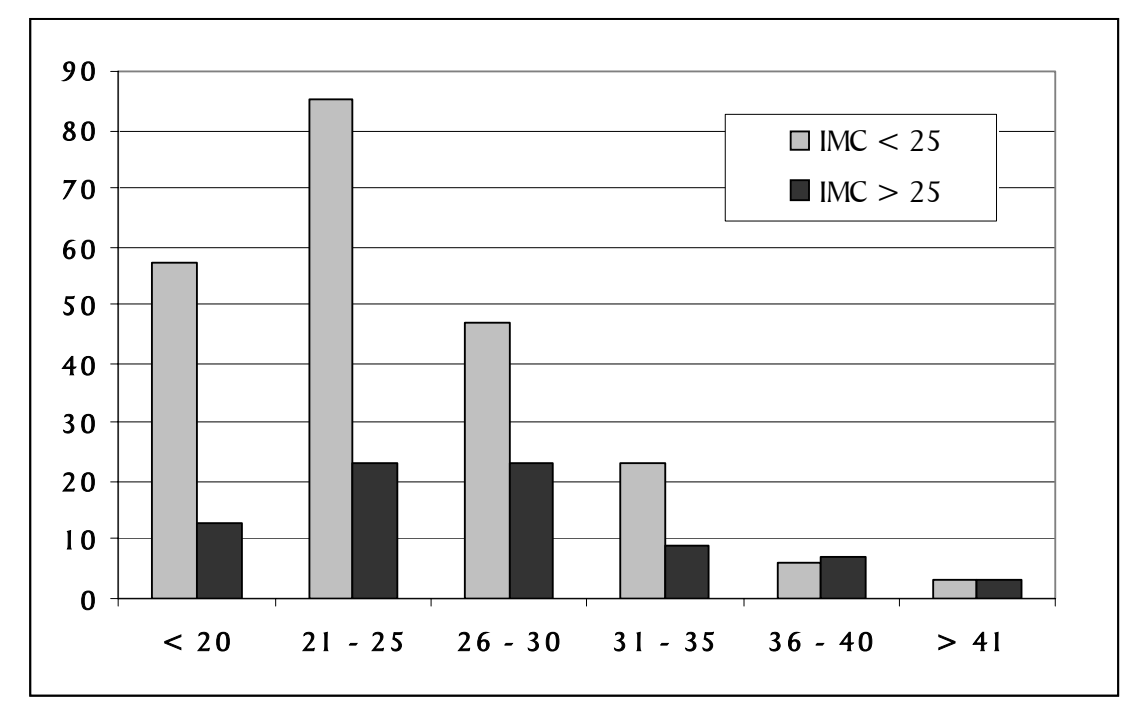

Figura 1. IMC de acordo com as faixas etárias. São Paulo, 2009.

Tabela 1. Estudantes de enfermagem com IMC $<25$ e $>25$, segundo gênero em relação à presença $(P)$ e ausência $(A)$ de HAS como fator de risco para DAC. São Paulo, 2009.

\begin{tabular}{|c|c|c|c|c|}
\hline \multirow{2}{*}{ Gênero } & \multicolumn{2}{|c|}{ IMC $<25$} & \multicolumn{2}{|c|}{ IMC $\geq 25$} \\
\hline & $\mathbf{P}$ & A & $\mathbf{P}$ & $A$ \\
\hline Feminino & 5 & 190 & 4 & 56 \\
\hline Masculino & 2 & 26 & 0 & 20 \\
\hline Total & 7 & 216 & 4 & 76 \\
\hline
\end{tabular}


Tabela 2. Relação da ocorrência simultânea dos fatores associados para DAC de acordo com IMC entre os estudantes de enfermagem. São Paulo, 2009.

\begin{tabular}{|c|c|c|c|c|c|c|c|c|}
\hline \multirow{3}{*}{ Fatores de Risco } & \multicolumn{4}{|c|}{ Feminino } & \multicolumn{4}{|c|}{ Masculino } \\
\hline & \multicolumn{2}{|c|}{ IMC $<25$} & \multicolumn{2}{|c|}{ IMC $\geq 25$} & \multicolumn{2}{|c|}{ IMC $<25$} & \multicolumn{2}{|c|}{ IMC $\geq 25$} \\
\hline & $\mathrm{n}$ & $\%$ & $\mathrm{n}$ & $\%$ & $\mathrm{n}$ & $\%$ & $\mathrm{n}$ & $\%$ \\
\hline Idade & 10 & 5,1 & 11 & 18,3 & - & 0,0 & 1 & 5,0 \\
\hline Raça & 17 & 8,7 & 4 & 3,7 & 11 & 39,3 & 3 & 15,0 \\
\hline HAS & 5 & 2,6 & 4 & 3,7 & 2 & 7,1 & - & - \\
\hline DM & 1 & 0,5 & - & - & - & - & - & - \\
\hline Atividade Física & 178 & 91,3 & 49 & 81,7 & 19 & 67,9 & 17 & 85,0 \\
\hline Tabagismo & 6 & 3,1 & 11 & 18,3 & 1 & 3,6 & 2 & 10,0 \\
\hline Estresse & 128 & 65,6 & 45 & 75,0 & 15 & 53,6 & 12 & 60,0 \\
\hline Padrão do Sono & 68 & 34,9 & 29 & 48,3 & 14 & 50,0 & 11 & 55,0 \\
\hline História Familiar & 39 & 20,0 & 17 & 28,3 & 7 & 25,0 & 4 & 20,0 \\
\hline Dislipidemia & 81 & 41,5 & 11 & 18,3 & 10 & 35,7 & 7 & 35,0 \\
\hline
\end{tabular}

Teste de $G$ de Cochran:

(Idade x Raça x HAS x DM x Atividade Física x Tabagismo x Estresse x Padrão do Sono x Histórico Familiar x Dislipidemia)

Tabela 3. Relação entre IMC e faixas etárias como associação de risco. São Paulo, 2009.

\begin{tabular}{|c|c|c|c|c|c|c|c|c|}
\hline \multirow{3}{*}{ Faixas Etárias } & \multicolumn{4}{|c|}{ IMC $<25$} & \multicolumn{4}{|c|}{ IMC $\geq 25$} \\
\hline & \multicolumn{2}{|c|}{ Feminino } & \multicolumn{2}{|c|}{ Masculino } & \multicolumn{2}{|c|}{ Feminino } & \multicolumn{2}{|c|}{ Masculino } \\
\hline & $\mathrm{n}$ & $\%$ & $\mathrm{n}$ & $\%$ & $n$ & $\%$ & $\mathrm{n}$ & $\%$ \\
\hline$\leq 20$ & 53 & 27,2 & 4 & 14,3 & 13 & 21,7 & - & - \\
\hline$\overline{21}-25$ & 76 & 39,0 & 10 & 35,7 & 13 & 21,7 & 10 & 50,0 \\
\hline $26-30$ & 37 & 19,0 & 10 & 35,7 & 15 & 25,0 & 8 & 40,0 \\
\hline $31-35$ & 19 & 9,7 & 4 & 14,3 & 8 & 13,3 & 1 & 5,0 \\
\hline $36-40$ & 7 & 3,6 & - & - & 8 & 13,3 & 1 & 5,0 \\
\hline$\geq 41$ & 3 & 1,5 & - & - & 3 & 5,0 & - & - \\
\hline Total & 195 & 100,0 & 28 & 100,0 & 60 & 100,0 & 20 & 100,0 \\
\hline
\end{tabular}

\section{DISCUSSÃO}

A partir dos resultados apresentados, os fatores de risco modificáveis são os mais significantes para o desenvolvimento de DAC entre os participantes do estudo. Dentre estes fatores, destacou-se o sobrepeso (IMC $\geq 25$ ). De acordo com a prevalência de obesidade, a mesma está relacionada a vários outros determinantes como fatores genéticos, consumo de açúcar, alimentação desequilibrada e falta de exercício físico ${ }^{(20)}$.

A obesidade pode ser desencadeada pela má alimentação. Neste caso, vale ressaltar que o fato de Que o estudante, dependendo do turno em Que estuda e de outras atividades desenvolvidas durante o dia, como o trabalho, tende ter prejudicado o seu hábito alimentar $^{(21,22)}$. Junta-se a isto o fato de Que o estudante ainda gasta muito tempo em seu deslocamento para a universidade, considerando as características do transporte metropolitano. Estes fatores corroboram o desenvolvimento da obesidade e merecem ser investigados em outros estudos.

A correlação/associação de fatores conduziu ao dado de maior significância deste estudo e está relacionado ao fator idade. O IMC $\geq$ 25 associado à faixa etária e gênero produziu o resultado $p=0,043$ para a faixa etária de 21 a 25 anos e $p=0,034$ para a faiza etária de 26 a 30 anos.No estudo, o resultado de maior representativiade entre todas as faixas etárias estabelecidas foi na de 2 I a 25 anos (36,0\%). Este resultado adQuire significância ${ }^{(8)}$.
De uma maneira geral, é necessário Que o estudante de enfermagem, profissional este em formação para a atividade de cuidar, precisa ser ensinado a cuidar-se. Kestemberg et al ${ }^{(23)}$, salientam Que é necessário Que o estudante saiba reconhecer os seus limites para um desenvolvimento mais equilibrado de si mesmo. É preciso reconhecer Que para se tornar um cuidador, antes é preciso saber cuidar-se. Este saber cuidar-se inclui a tomada de decisão com respeito às Questões individuais de saúde Que incluem uma alimentação equilibrada, abstenção de substâncias nocivas à saúde, observância de horas de sono, realização de atividades físicas, etc.

Sobre os fatores de risco isolados de maior significância, ressaltou-se a falta de realização de atividade física entre 76,9\% dos estudantes. Apenas 19,8\% dos estudantes realizam regularmente a atividade física. $\mathrm{O}$ sedentarismo é um dos fatores de risco Que está diretamente relacionado a outros fatores como o estresse $^{(15,24)}$, obesidade ${ }^{(21)}$ e $\operatorname{HAS}^{(7)}$.

\section{CONCLUSÃO}

Este estudo identificou os fatores de risco associados a DAC entre estudantes de enfermagem. Os fatores associados de maior significância foram: IMC $\geq 25$ na faixa etária de 21 a 25 e de 26 a 30 anos em indivíduos do gênero masculino (Teste do Qui-Quadrado $p=0,043$ e $p=0,03$ I respectivamente). O fator de risco isolado de maior prevalência foi o sedentarismo. 
Os estudantes de enfermagem, em geral, têm a visão voltada à vida acadêmica e as responsabilidades com datas e horários. Isto tudo pode ter relação com a transformação do cotidiano dos estudantes em uma corrida contra o tempo em Que a praticidade como transporte, alimentos industrializados, e cuidados com a própria saúde fieuem relegados a um segundo plano. A ausência de sintomas significativos de DAC, ou mesmo o não seguimento de medidas preventivas como a prática de atividades físicas e alimentação balanceada podem ter um peso nos resultados encontrados neste estudo. Estes resultados levam à reflexão de Que intervenções devam ser implementadas como medidas preventivas de DAC.

Novos estudos são necessários para evidenciar as causas do sobrepeso entre os estudantes de enfermagem. É importante Que haja a conscientização de Que Quanto melhor for o controle dos hábitos de vida com redução do número de fatores modificáveis associados, maior é a redução dos riscos associados a DAC.

\section{REFERÊNCIAS}

I. Porto CC, Porto AL. Doença do coração: prevenção e tratamento. 2a ed. Rio de Janeiro: Guanabara Koogan; 2005.

2. World Health Organization. Health topics. Geneve; 2005. [cited 4 jun 2009]. Available from: http://www.who.int/ cardiovascular_diseases/en/

3. Falk E. Plaque ruptures with severe pre-existing stenosis precipitating thrombosis: characteristics of coronary atherosclerotic plaques underlying fatal occlusive thrombi. $\mathrm{Br}$ Heart I 1983; 50: 127-34.

4. Holman RL, McGill Jr HC, Strong JP, Geer IC. The natural history of atherosclerosis. Am J Pathol 1958; 34:209-35.

5. Stary HC. The sequence of cell and matrix changes in atherosclerotic lesions of coronary arteries in the first forty years of life. Eur Heart I 1990; I I (sup) E3-19.

6. Dórea EL, Lotufo PA. Framingham Heart Study e a teoria do Contínuo de Pickering. Rev Bras Hipertens 1992; 8:195-200.

7. Caramelli B; Ballas D; Ramires JAF. Doença Coronária e Aterosclerose: clínica, terapia intensiva e emergências. São Paulo: Atheneu; 1998.

8. World Health Organization. Noncommunicable diseases and mental health, integrated management of cardiovascular risk: report of a WHO meeting. Geneva 9-12, July 2002. Geneva: World Health Organization; 2002.

9. Markay MGA. The atlas of disease and stroke. Geneva: World Health Organization; 2004.

10. Fernandes CE, Pinho Neto ISL, Gebara OCE. I Diretriz Brasileira sobre Prevenção de Doença Cardiovasculares em Mulheres Climatéricas e a Influência da Terapia de Reposição Hormonal da Sociedade Brasileira de Cardiologia e da Associação Brasileira do Climatério. Are Bras Cardiol 2008; 91 (I sup I): 1-23.

1 1. World Health Organization. Building blocks for tobacco control. Geneva: World Health Organization; 2004.

12. Castelli WP, Garrison RJ, Wilson PWF, Abbott RD, Kalousdian $S$, Kannel WB. Incidence of coronary heart disease and lipoprotein cholesterol levels. The Framingham Study. JAMA 1986; 256: 2835-8.

13. Menotti A, Lanti M, Puddu PE, Kromhout D. Coronary heart disease incidence in northern and southern European population: a reanalysis of seven countries study for a European coronary risk chart. Heart 2000; 84: 238-44.

14. Lessa I. Assistência médica e óbitos por Doença Arterial Coronariana no Brasil, 1980-1999. Are Bras Cardiol 2003; 81(4): 329-35.

15. Loures DL, Sant'Anna I, Baldotto CSR, Sousa EB, Nóbrega ACL. Estresse mental e sistema cardiovascular. Are Bras Cardiol 2002; 75(5): 525-30.

16. Coelho VG, Caetano LF, Liberatore Júnior R del R, Cordeiro IA, Souza DRS. Perfil lipídico e fatores de risco para Doenças Cardiovasculares em estudantes de medicina. ArQ Bras Cardiol 2005; 85(I) 57-62.

17. Fisberg RM, Stella RH, Morimoto IM, Pasquali LS, Philippi ST, Latorre MRDO. Perfil lipídico de estudantes de nutrição e a sua associação com fatores de risco para doenças cardiovasculares. Are Bras Cardiol 200 I; 76(2): 137-42.

18. LoBiondo-Wood G, Haber I. Pesquisa em enfermagem: métodos, avaliação crítica e utilização. Rio de Janeiro: Guanabara Koogan; 2001 .

19. Siegel SE, Castellan Júnior NJ. Estatística não-paramétrica para ciências do comportamento. Porto Alegre: Artmed; 2006.

20. Gigante DP, Barros FC, Post CLA, Olinto MTA. Prevalência de obesidade em adultos e seus fatores de risco. Rev Saúde Pública 1997; 3 I (3): 236-46.

21. Saupe R, Nietche EA, Cestari ME, Glorgi MDM, Krahl M. Qualidade de vida dos acadêmicos de enfermagem. Rev Latinoam Enfermagem 2004; I2(4): 638-42.

22. Kawakame PMG, Miyadahira AMK. Qualidade de vida de estudantes de graduação em enfermagem. Rev Esc Enferm USP 2005; 39(2): 164-72.

23. Kestemberg CCF, Reis MMSA, Motta WC, Caldas MF, Rodrigues DMC. Cuidando do estudante e ensinando relações de cuidado de enfermagem. Texto Contexto Enferm 2006; 15(esp): 193-200.

24. Telles Filho PCP, Pires E, Araújo GA. Características evidenciáveis de estresse em discentes de enfermagem. Rev Latino-am Enfermagem 1999; 7(2): 91-3. 